\title{
Memory impairment at initial clinical presentation in posterior cortical atrophy
}

Samrah Ahmed ${ }^{\mathrm{a}}$, Ian Baker ${ }^{\mathrm{b}}$, Masud Husain ${ }^{\mathrm{a}, \mathrm{d}}$, Sian Thompson ${ }^{\mathrm{c}}$, Christopher Kipps ${ }^{\mathrm{e}}$, Michael Hornberger ${ }^{\mathrm{f}}$, John R. Hodges ${ }^{\mathrm{g}}$, Christopher R. Butler ${ }^{\mathrm{a}}$

${ }^{a}$ Nuffield Department of Clinical Neurosciences, University of Oxford, John Radcliffe Hospital, Oxford UK.

${ }^{\mathrm{b}}$ Russell Cairns Unit, Oxford University Hospitals NHS Trust, John Radcliffe Hospital, Oxford UK.

${ }^{\mathrm{c}}$ Department of Clinical Neurology, Oxford University Hospitals NHS Trust, Oxford, UK.

${ }^{\mathrm{d}}$ Department of Experimental Psychology, University of Oxford, John Radcliffe Hospital, Oxford UK.

${ }^{\mathrm{e}}$ Wessex Neurological Centre, University Hospitals Southampton NHS Foundation Trust and University of Southampton and Wessex Collaboration for Leadership in Applied Health Research and Care (CLAHRC).

${ }^{\mathrm{f}}$ Norwich Medical School, University of East Anglia, Norwich, NR4 7QU, UK.

${ }^{\mathrm{g}}$ Neuroscience Research Australia and University of New South Wales, Randwick, Sydney, Australia.

\section{Corresponding author:}

Dr Samrah Ahmed

Nuffield Department of Clinical Neurosciences

University of Oxford

John Radcliffe Hospital

Oxford OX3 9DU

United Kingdom

Email: samrah.ahmed@nden.ox.ac.uk 
Running title: Memory in posterior cortical atrophy

Keywords: Early onset Alzheimer's Disease; diagnosis; memory; neuropsychology. 


\section{Abstract}

Posterior cortical atrophy (PCA) is characterized by core visuospatial and visuoperceptual deficits, and predominant atrophy in the parieto-occipital cortex. The most common underlying pathology is Alzheimer's disease. Existing diagnostic criteria suggest that episodic memory is relatively preserved. The aim of this study was to examine memory performance at initial clinical presentation in PCA, compared to early-onset AD patients (EOAD). 15 PCA patients and $32 \mathrm{EOAD}$ patients, and 34 healthy controls were entered into the study. Patients were tested on the Addenbrooke's Cognitive Examination (ACE-R), consisting of subscales in memory and visuospatial skills. PCA and EOAD patients were significantly impaired compared to controls on the ACE total score $(\mathrm{p}<.001)$, visuospatial skills $(\mathrm{p}<.001)$ and memory $(\mathrm{p}<.001)$. Consistent with the salient diagnostic deficits, PCA patients were significantly more impaired on visuospatial skills compared to EOAD patients $(p<.001)$. However, there was no significant difference between patient groups in memory. Further analysis of learning, recall and recognition components of the memory subscale showed that EOAD and PCA patients were significantly impaired compared to controls on all three components $(\mathrm{p}<.001)$, however, there was no significant difference between EOAD and PCA patients. The results of this study show that memory is impaired in the majority of PCA patients at clinical presentation. The findings suggest that memory impairment must be considered in assessment and management of PCA and second. Further study into memory in PCA is warranted, since the ACE-R is a brief screening tool and is likely to underestimate the presence of memory impairment. 


\section{Introduction}

Proposed diagnostic criteria for posterior cortical atrophy (PCA) [14, 20] emphasize core visuospatial and visuoperceptual deficits, not attributable to ocular disease. Predominant atrophy is in the parieto-occipital cortex, with relative sparing of the temporal regions. The most common underlying pathology is Alzheimer's disease (AD) $[1,9,18]$. Episodic memory is thought to be relatively preserved in PCA. It is suggested that anterograde memory is not impaired in the early stages, but gradually deteriorates with progression of disease $[14,15]$. As a result, memory has rarely been examined systematically in PCA. Where memory assessments have been reported, the findings have been variable. Comparing PCA with typical AD (tAD), in which the hallmark feature is one of early and profound episodic memory impairment, McMonagle et al. [14] found that PCA patients scored lowest on measures of visuoconstruction, as expected, but in addition there was no significant difference compared with tAD on measures of memory. PCA patients achieved less than half the maximum score on memory testing, similar to tAD performance. Similarly, Charles and Hillis [4] found that memory, as assessed by a verbal list learning task, was impaired in both PCA and tAD patients, although tAD patients showed greater impairment on delayed recall. Conversely, Kas et al. [10] found that PCA patients performed significantly better than tAD patients on an episodic verbal memory test, on both free and cued recall components. The variable findings could be due to differences in disease duration, however Kas et al. [10] divided PCA patients into short ( $\leq 3$ years) and long ( $>3$ years) disease duration groups, and reported no significant differences in neurological or neuropsychological symptoms between the two PCA groups. Each of these studies also administered different memory tests, and in different sample sizes, which may also explain the discrepant findings. 
Misdiagnosis in PCA is common due to the relative rarity of presentation and unusual symptoms. Patients frequently describe a plethora of referrals and procedures undertaken by opticians and opthalmologists in the belief that an ocular abnormality accounts for the visual deficits experienced. Equally common are patients presenting with an accompanying mood disorder which is then wrongly considered to be the primary diagnosis [5]. The presence of mild or even more prominent memory impairment early in PCA may assist in the recognition and diagnosis of PCA and prevent delays in diagnosis.

The aim of this study was to examine memory performance in PCA patients at initial clinical presentation to a cognitive disorders clinic, compared to early-onset AD (EOAD) patients with a typical memory impaired presentation.

\section{Materials and methods}

\section{Participants}

All patients were assessed at clinical presentation at the Oxford Cognitive Disorders Clinic, Oxford, UK; Wessex Cognitive Disorders Clinic, UK, or the Frontotemporal Dementia Clinic, Sydney, Australia. A total of 47 patients were entered into the study: 15 with PCA and 32 with EOAD. Patient performance was compared to 34 healthy controls. Healthy controls were all recruited from the Frontotemporal Dementia Clinic, Sydney, Australia. All patients were assessed by a senior behavioural neurologist (CB, ST, MH, CK or JRH). PCA patients fulfilled available consensus criteria $[14,20]$ : patients displayed any of the core features of simultanagnosia with or without optic ataxia or ocular apraxia, constructional apraxia, visual field defect, 
elements of Gerstmann's syndrome or environmental disorientation, with any of the supportive features of alexia, ideomotor or dressing apraxia, or prosopagnosia. EOAD [13] patients were all under the age of 65 at presentation. Patients underwent either an MRI or CT scan where MR was contraindicated (e.g. by a cardiac pacemaker), within 6 months of presentation. Imaging results were consistent with diagnosis. The study was approved by the South Eastern Sydney and Illawarra Area Health Service and the University of New South Wales human ethics committees, the National Research Ethics Service South Central - Hampshire B and Oxford C. All participants or their Person Responsible provided written informed consent in accordance with the Declaration of Helsinki. The genetic status of all patients was unknown.

\section{Cognitive testing}

Patients were tested on the Addenbrooke's Cognitive Examination-Revised (ACE-R; [16]), a widely used screening tool that is administered to all patients at presentation to the memory clinics. The ACE-R incorporates tests of memory, attention and orientation, language, fluency and visuospatial skills. The memory sub-score of the ACE-R consists of learning of a name and address and recall and recognition of this information approximately 15 minutes later. There are also questions testing shortterm memory by asking participants to recall three words, and remote memory testing recall to description of the names of famous politicians.

\section{Statistical analysis}

Normality of distribution of the data was examined using Shapiro-Wilk tests. Group differences in demographic, clinical and cognitive characteristics were examined using one-way analysis of variance, or with Kruskal-Wallis with pairwise 
comparisons performed using Mann-Whitney tests where assumptions for parametric tests were not met. An independent sample t-test was used to examine differences in symptom duration. Two-tailed tests were conducted with alpha level set at 0.05 .

\section{Results}

Demographic and clinical characteristics

The demographic and clinical characteristics of patients are shown in Table 1. There were no significant differences in age or education. Patients were matched on symptom duration.

-Table 1 here-

\section{Memory and visuospatial scores}

Results of group comparisons on the ACE subscales are given in Table 2 and graphically represented in Figure 1. Both PCA and EOAD patients were significantly impaired compared to controls on the ACE total scores $(\mathrm{p}<.001)$, on visuospatial skills $(\mathrm{p}<.001)$ and on memory $(\mathrm{p}<.001)$.

There was no significant difference between patient groups on ACE total score $(p=.262)$. As expected, PCA patients were significantly more impaired on visuospatial skills compared to EOAD patients $(\mathrm{p}<.001)$, in accordance with the salient diagnostic deficits in this group. However, there was no significant difference between patient groups $(\mathrm{p}=.619)$ on the memory subscale. Figure 1 shows that the range of memory scores was similar in PCA and EOAD, patients with the majority of both patient 
groups scoring below the control range. Visuospatial scores were consistently low in PCA patients compared with controls, but more variable in EOAD.

-Table 2 here-

-Figure 1 here-

Further analysis was conducted of the main constituents of the ACE-R memory score, in a subset of the patient $(n=30)$ and control $(n=34)$ sample for whom data was available. This consists of learning a name and address, recalling the information after delay (typically 10 minutes, depending on the speed of the patient), and finally a recognition test of any items not recalled. Table 3 shows that both EOAD and PCA patients were significantly impaired compared with controls on all three of the memory components $(\mathrm{p}<.001)$, however, there was no significant difference between EOAD and PCA patients.

-Table 3 here-

\section{Clinical profile of PCA patients}

Further data from each of the 15 PCA patients at initial presentation are shown in Table 4. Clinical characteristics were consistent with the core features of PCA [20]. Memory impairment (based on the ACE-R memory sub-score and classed as 2+ standard deviations below the control mean) was evident in 10 of the 15 PCA patients assessed (66.7\%). There was no significant difference between these memory impaired PCA patients and those with preserved memory, in terms of age, education 
or disease duration. Of the PCA patient with memory impairment, scores for 9 out of 10 of PCA patients (90\%) fell within the range of EOAD scores.

-Table 4 here-

\section{Discussion}

Diagnostic criteria for PCA have emphasized salient visual deficits in the absence of ocular disease, and preserved or only mildly impaired episodic memory.

Consequently, memory has rarely been systematically examined in PCA and the few studies that have included memory assessment report variable findings [4, 10, 14]. The results of this study show that memory is often impaired in PCA patients at clinical presentation. $66.7 \%$ of PCA patients had impaired memory compared to healthy controls, suggesting that early memory impairment is common, although not ubiquitous in PCA. Furthermore, while PCA patients could be characterized by salient and significant deficits in visuospatial features compared to controls and EOAD patients, memory was also impaired in PCA. The mean scores were indistinguishable between the two patient groups. Early impairment in memory alongside the defining visual disturbances, is consistent with the handful of past studies that have included simple memory measure in the neuropsychological assessment $[4,14]$. However, the reasons for impairment are not clear. We propose three possible explanations.

Firstly, impairment may be driven by the primary pathology in the parietal cortex, specifically affecting attentional networks underlying memory. In task-based neuroimaging studies, the posterior parietal cortices are frequently activated in response to episodic memory retrieval [3]. A meta-analytic review [19] identified the 
parietal lobes as being more consistently activated during recollection, identified in $90 \%$ of studies reviewed, compared to more typically considered episodic memory regions such as the medial temporal lobe. Neuropsychological studies of patients with bilateral parietal lobe lesions have found that patients are impaired on tests of recollection, such as autobiographical memory retrieval [2] or remember/know paradigms [7], and provide few 'remembered' responses, and lack of specificity and richness of freely recalled autobiographical events.

Secondly, PCA patients may have more widespread pathology extending to medial temporal memory-related structures. Tang-Wai et al. [20] described a group of PCA patients in detail to form the basis of currently used diagnostic criteria, and found relatively preserved medial temporal lobe structures, which could be appreciated antemortem on MRI scans. Conversely, Migliaccio et al. [15] found that regions of grey matter reduction in PCA included the hippocampus bilaterally. Studies examining cortical thickness patterns have also reported reductions in the medial temporal lobe [12]. The majority of our patients presented within 2-3 years of symptom onset, and imaging was consistent with salient parieto-temporal atrophy. However, more detailed imaging analysis may have highlighted more extensive deficits.

A third possibility is that verbal memory impairment may be attributable to disruption of language networks in the left hemisphere. A recent study described subtle language impairments in PCA characterized by anomia, poor phonemic fluency and slow speech rate, resembling the linguistic profile most consistent with logopenic aphasia [6]. Language was not assessed in detail at clinical presentation in the PCA 
patients reported in this study. However, it is worth noting the PCA patients assessed in Crutch et al. [6] had an average symptom duration of 6 years, more than twice that of the majority of PCA patients in this study, and thus it is suggested that language impairment may be a feature of more advanced stages of PCA.

A small percentage of PCA patients scored within the control range. These patients were comparable to the impaired PCA group in age, education and, importantly, in symptom duration. Preserved memory in this small group may be due to individual variation in disease expression, or due to the insensitivity of the ACE-R to subtle memory impairment.

There are limitations to this study. The ACE-R is a brief screening tool and was used to demonstrate that memory impairment can be detected in PCA with a broad measure at presentation. The ACE-R is likely therefore to underestimate the extent of memory impairment. It is also unable to provide detailed information as to the nature of episodic memory impairment in PCA and EOAD. Further investigation with detailed neuropsychological testing of memory function in PCA is therefore essential. However, the results support the conclusion that memory impairment can be detected at initial presentation with a simple screening instrument, and with a relatively short average symptom duration compared to other studies (for example, $[6,10]$ ). Despite differences in the predominant clinical symptoms in PCA and EOAD, the concomitant and significant memory impairment early in the clinical course may reflect the common underlying $\mathrm{AD}$ pathology. A similar finding has been reported in an earlier study, examining patients with logopenic aphasia (lv-PPA), characterized by impaired repetition of sentences and naming and in which the underlying 
pathology is $\mathrm{AD}$ in the majority of patients. Flanagan et al. [8] found that lv-PPA patients were significantly impaired in memory, and did not differ from a tAD group, suggesting that identifying specific impairments in memory may supplement diagnosis of patients with pathology.

These findings have clear clinical implications. Current diagnostic criteria emphasize the relative preservation of memory in PCA, yet the findings provide evidence of a detectable memory deficit early in the course of disease. Further study is warranted to determine how diagnostic criteria may need to be revised. Diagnostically, the presence of memory impairment may assist in the early identification of PCA, helping to differentiate from visual disturbances caused by ocular or other problems. Improving our understanding of more focal presentations of $\mathrm{AD}$ is imperative to facilitate accurate prediction of AD pathology in life, and support the development of target therapies for AD. Studies suggest that variant clinical presentations of AD are not so infrequent, representing a third of patients with early-onset $\mathrm{AD}$, and at least $5 \%$ of late onset cases [11]. In terms of intervention and management, while it is entirely appropriate to centre care and advice on the critical visual impairments experienced by patients, it is also important to consider problems arising from memory impairment. The literature on tAD cites extensive examples of the pervasive difficulties memory impairment incites, e.g. in restricting activities of daily living [17]. Memory impairment therefore must be considered in assessment and management of PCA. 


\section{Acknowledgements}

We are indebted to the patients and families for their kind participation in our research. Our thanks to Lesley MacKinnon for her assistance with data collection. SA is supported by the NIHR Oxford Biomedical Research Centre and Alzheimer's Research UK. M. Hornberger is supported by Alzheimer's Research UK, Medical Research Council and Wellcome Trust. M. Husain is supported by the Wellcome Trust. CB is supported by the Medical Research Council (MR/K010395/1). This work was supported by funding to Forefront a collaborative research group dedicated to the study of frontotemporal dementia and motor neurone disease from the National Health and Medical Research Council of Australia (\#1037746) and the Australian Research Council Centre of Excellence in Cognition and its Disorders Memory Node (\#CE110001021). There are no conflicts of interest to report. 


\section{References}

[1] Alladi S, Xuereb J, Bak T, Nestor P, Knibb J, Patterson K, Hodges JR (2007) Focal cortical presentations of Alzheimer's disease. Brain 130, 26362645.

[2] Berryhill ME, Phuong L, Picasso L, Cabeza R, Olson IR (2007) Parietal lobe and episodic memory: bilateral damage causes impaired free recall of autobiographical memory. J Neurosci 27, 14415-14423.

[3] Cabeza R, Nyberg L (2000) Neural bases of learning and memory: functional neuroimaging evidence. Curr Opin Neurol 13, 415-421.

[4] Charles RF, Hillis AE (2005) Posterior cortical atrophy: clinical presentation and cognitive deficits compared to Alzheimer's disease. Behav Neurol 16, 15-23.

[5] Crutch SJ, Lehmann M, Schott JM, Rabinovici GD, Rossor MN, Fox NC (2012) Posterior cortical atrophy. Lancet Neurol 11, 170-178.

[6] Crutch SJ, Lehmann M, Warren JD, Rohrer JD (2013) The language profile of posterior cortical atrophy. J Neurol Neurosurg Psychiatry 84, 460-466.

[7] Davidson PS, Anaki D, Ciaramelli E, Cohn M, Kim AS, Murphy KJ, Troyer AK, Moscovitch M, Levine B (2008) Does lateral parietal cortex support episodic memory? Evidence from focal lesion patients. Neuropsychologia 46, 1743-1755.

[8] Flanagan EC, Tu S, Ahmed S, Hodges JR, Hornberger M (2014) Memory and orientation in the logopenic and nonfluent subtypes of primary progressive aphasia. J Alzheimers Dis 40, 33-36.

[9] Galton CJ, Patterson K, Xuereb JH, Hodges JR (2000) Atypical and typical presentations of Alzheimer's disease: a clinical, neuropsychological, neuroimaging and pathological study of 13 cases. Brain 123 Pt 3, 484498.

[10] Kas A, de Souza LC, Samri D, Bartolomeo P, Lacomblez L, Kalafat M, Migliaccio R, Thiebaut de Schotten M, Cohen L, Dubois B, Habert MO, Sarazin M (2011) Neural correlates of cognitive impairment in posterior cortical atrophy. Brain 134, 1464-1478.

[11] Koedam EL, Lauffer V, van der Vlies AE, van der Flier WM, Scheltens P, Pijnenburg YA (2010) Early-versus late-onset Alzheimer's disease: more than age alone. J Alzheimers Dis 19, 1401-1408.

[12] Lehmann M, Rohrer JD, Clarkson MJ, Ridgway GR, Scahill RI, Modat M, Warren JD, Ourselin S, Barnes J, Rossor MN, Fox NC (2010) Reduced cortical thickness in the posterior cingulate gyrus is characteristic of both typical and atypical Alzheimer's disease. J Alzheimers Dis 20, 587-598.

[13] McKhann G, Drachman D, Folstein M, Katzman R, Price D, Stadlan EM (1984) Clinical diagnosis of Alzheimer's disease: report of the NINCDSADRDA Work Group under the auspices of Department of Health and Human Services Task Force on Alzheimer's Disease. Neurology 34, 939944.

[14] McMonagle P, Deering F, Berliner Y, Kertesz A (2006) The cognitive profile of posterior cortical atrophy. Neurology 66, 331-338.

[15] Migliaccio R, Agosta F, Rascovsky K, Karydas A, Bonasera S, Rabinovici GD, Miller BL, Gorno-Tempini ML (2009) Clinical syndromes associated with 
posterior atrophy: early age at onset AD spectrum. Neurology $\mathbf{7 3}, 1571$ 1578.

[16] Mioshi E, Dawson K, Mitchell J, Arnold R, Hodges JR (2006) The Addenbrooke's Cognitive Examination Revised (ACE-R): a brief cognitive test battery for dementia screening. International journal of geriatric psychiatry 21, 1078-1085.

[17] Mioshi E, Kipps CM, Dawson K, Mitchell J, Graham A, Hodges JR (2007) Activities of daily living in frontotemporal dementia and Alzheimer disease. Neurology 68, 2077-2084.

[18] Renner JA, Burns JM, Hou CE, McKeel DW, Jr., Storandt M, Morris JC (2004) Progressive posterior cortical dysfunction: a clinicopathologic series. Neurology 63, 1175-1180.

[19] Simons JS, Peers PV, Hwang DY, Ally BA, Fletcher PC, Budson AE (2008) Is the parietal lobe necessary for recollection in humans? Neuropsychologia 46, 1185-1191.

[20] Tang-Wai DF, Graff-Radford NR, Boeve BF, Dickson DW, Parisi JE, Crook R, Caselli RJ, Knopman DS, Petersen RC (2004) Clinical, genetic, and neuropathologic characteristics of posterior cortical atrophy. Neurology 63, 1168-1174. 


\begin{tabular}{|c|c|c|c|c|}
\hline & $\begin{array}{c}\text { Normal } \\
\text { controls } \\
(\mathrm{n}=34) \\
\end{array}$ & $\begin{array}{l}\text { EOAD } \\
(\mathrm{n}=32)\end{array}$ & $\begin{array}{c}\text { PCA } \\
(\mathrm{n}=15)\end{array}$ & $p$ \\
\hline Age at onset & $\begin{array}{l}59.8 \\
(3.9)\end{array}$ & $\begin{array}{l}57.5 \\
(5.3)\end{array}$ & $\begin{array}{l}59.5 \\
(6.9)\end{array}$ & n.s. \\
\hline Education & $\begin{array}{l}13.7 \\
(2.9)\end{array}$ & $\begin{array}{l}13.4 \\
(2.4)\end{array}$ & $\begin{array}{l}12.3^{*} \\
(2.0)\end{array}$ & n.s. \\
\hline Gender (m:f) & $17: 17$ & $15: 17$ & $7: 8$ & - \\
\hline $\begin{array}{l}\text { Symptom duration } \\
\text { (yrs) }\end{array}$ & - & $\begin{array}{c}2.6 \\
(1.2)\end{array}$ & $\begin{array}{c}2.4 \\
(0.86)\end{array}$ & n.s. \\
\hline
\end{tabular}

Table 1. Demographic and clinical characteristics of patient groups and controls. 


\begin{tabular}{lcccc}
\hline & $\begin{array}{c}\text { Normal } \\
\text { controls } \\
(\mathrm{n}=34)\end{array}$ & $\begin{array}{c}\text { EOAD } \\
(\mathrm{n}=32)\end{array}$ & $\begin{array}{c}\text { PCA } \\
(\mathrm{n}=15)\end{array}$ & $\begin{array}{c}\text { Omnibus } \\
\boldsymbol{p}\end{array}$ \\
\hline ACE-R Memory (26) & 24.3 & $13.2^{\mathrm{a}}$ & $16.1^{\mathrm{a}}$ & $<.001$ \\
& $(1.9)$ & $(5.0)$ & $(6.0)$ & \\
\hline ACE-R Visuospatial (16) & 15.4 & $10.9^{\mathrm{a}, \mathrm{b}}$ & $7.1^{\mathrm{a}}$ & $<.001$ \\
& $(1.0)$ & $(4.0)$ & $(3.7)$ & \\
\hline ACE-R Total (100) & 94.7 & $65.5^{\mathrm{a}}$ & $66.4^{\mathrm{a}}$ & $<.001$ \\
& $(3.1)$ & $(15.1)$ & $(12.7)$ & \\
\hline ACE-R Addenbrooke's Cognitive Examination-Revised; EOAD Early onset AD; PCA & & \\
Posterior cortical atrophy \\
'aignificant difference compared to controls $p<.001$ \\
${ }^{b}$ Significant difference compared to PCA $p<.001$
\end{tabular}

Table 2. Group scores on the Addenbrookes's Cognitive Examination-Revised (ACE$\mathrm{R})$. 


\begin{tabular}{|c|c|c|c|c|}
\hline & $\begin{array}{c}\text { Normal } \\
\text { controls } \\
(n=34)\end{array}$ & $\begin{array}{r}\text { EOAD } \\
(\mathrm{n}=14)\end{array}$ & $\begin{array}{c}\text { PCA } \\
(\mathrm{n}=16)\end{array}$ & $\begin{array}{c}\text { Omnibus } \\
p\end{array}$ \\
\hline ACE-R learning (7) & $\begin{array}{c}7.0 \\
(0.1)\end{array}$ & $\begin{array}{l}4.9^{\mathrm{a}} \\
(2.2)\end{array}$ & $\begin{array}{l}5.4^{\mathrm{a}} \\
(1.7)\end{array}$ & $<.001$ \\
\hline ACE-R recall (7) & $\begin{array}{c}5.9 \\
(1.3)\end{array}$ & $\begin{array}{l}1.4^{\mathrm{a}} \\
(1.7)\end{array}$ & $\begin{array}{l}1.9^{\mathrm{a}} \\
(2.5)\end{array}$ & $<.001$ \\
\hline ACE-R recognition (5) & $\begin{array}{c}4.7 \\
(0.5)\end{array}$ & $\begin{array}{l}3.6^{\mathrm{a}} \\
(0.6)\end{array}$ & $\begin{array}{l}3.6^{\mathrm{a}} \\
(1.1)\end{array}$ & $<.001$ \\
\hline
\end{tabular}

Table 3. Group scores on the ACE-R memory questions 


\begin{tabular}{|c|c|c|c|c|c|c|c|c|c|c|c|c|c|c|c|}
\hline PCA patient & 1 & 2 & 3 & 4 & 5 & 6 & 7 & 8 & 9 & 10 & 11 & 12 & 13 & 14 & 15 \\
\hline \multicolumn{16}{|l|}{ Demographics } \\
\hline Age at onset (yrs) & 51 & 76 & 63 & 55 & 58 & 57 & 61 & 54 & 63 & 56 & 63 & 48 & 57 & 65 & 66 \\
\hline Gender & $\mathrm{F}$ & $\mathrm{M}$ & $\mathrm{F}$ & $\mathrm{F}$ & $\mathrm{M}$ & $\mathrm{M}$ & $\mathrm{M}$ & $\mathrm{F}$ & $\mathrm{F}$ & $\mathrm{M}$ & $\mathrm{M}$ & $\mathrm{F}$ & $\mathrm{F}$ & $\mathrm{M}$ & $\mathrm{F}$ \\
\hline Symptom duration (yrs) & 2.0 & 2.5 & 2.0 & 1.0 & 1.2 & 3.0 & 2.0 & 2.5 & 2.0 & 2.0 & 4.0 & 1.8 & 2.5 & 3.0 & 4.0 \\
\hline ACE Total & 76 & 78 & 54 & 69 & 63 & 76 & 55 & 75 & 78 & 79 & 85 & 53 & 51 & 45 & 59 \\
\hline \multicolumn{16}{|l|}{$\begin{array}{c}\text { Core features (Tang-Wei et al. 2004) } \\
\text { Any of the following: }\end{array}$} \\
\hline $\begin{array}{l}\text { Simultanagnosia with or without optic } \\
\text { ataxia or ocular apraxia }\end{array}$ & & + & + & + & & & & & + & + & + & + & + & + & + \\
\hline Constructional apraxia & + & & & & + & + & + & + & + & & + & + & + & + & + \\
\hline Visual field defect & + & + & & & + & + & & + & & & & & & + & + \\
\hline Elements of Gerstmann's syndrome & + & & & + & + & + & + & & & + & & & & & \\
\hline Environmental disorientation & & + & & & & & & & & & & & & & \\
\hline \multicolumn{16}{|l|}{$\begin{array}{c}\text { Supportive features (Tang-Wei et al. } \\
\text { 2004): }\end{array}$} \\
\hline Alexia & + & + & & & & & + & + & & & & + & & + & \\
\hline Ideomotor or dressing apraxia & & & + & + & + & & + & & & & + & + & & + & + \\
\hline Prosopagnosia & & & & & & & & & & & & & + & & \\
\hline Imaging consistent with PCA & + & + & + & + & + & + & + & + & + & + & + & + & + & + & + \\
\hline \multicolumn{16}{|l|}{ Memory } \\
\hline $\begin{array}{l}\text { Memory impairment (based on ACE-R } \\
\text { memory subscore) }\end{array}$ & - & + & + & + & + & - & + & + & - & - & - & + & + & + & + \\
\hline
\end{tabular}

Table 4. Individual case analysis of 15 PCA patients. 

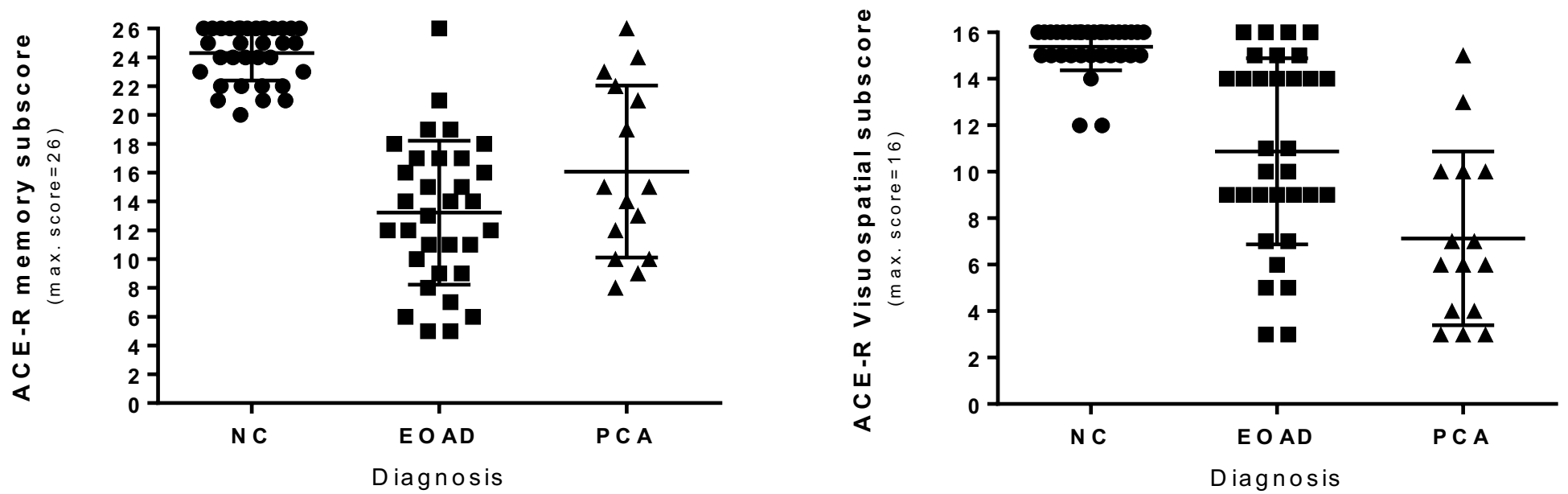

Figure 1. Scatterplots representing individual ACE-R memory and visuospatial scores for controls (NC), early onset AD (EOAD) and PCA (posterior cortical atrophy) patients. 